\title{
Effect of low-protein diet supplemented with short- and medium chain fatty acids on immune responses of $E$. coli challenged weaned piglets
}

\author{
M. Sugiharto, S. Hedemann, B. B. Jensen and C. Lauridsen, \\ Department of Animal Science, Faculty of Science and Technology, Aarhus University, AU-Foulum, Tjele, Denmark
}

To maintain a healthy intestine and support the immunity of young pigs, nutritional strategies have been formulated by means of lowering dietary protein ${ }^{(1)}$ or supplementing the diets with selected fatty acids ${ }^{(2)}$. However, little is known about the impact of these combined strategies on the immune responses of piglets. Using 24 newly weaned piglets ( 28 days of age, 7.68 [SD 1.72 ] $\mathrm{kg}$ body weight, tested susceptible to $E$. coli F4), a $2 \times 2$ factorial study was carried out in two consecutive trials to determine the effect of low-protein diet supplemented with short- and medium chain fatty acids on immune responses of weaned piglets challenged with $E$. coli. Twelve piglets received low-crude protein $(\mathrm{CP}, 20 \%)$ diet supplemented with butyric (4: 0, 0.29\%), caproic (6: 0, 0.15\%) and lauric acid (12: 0, 0.26\%), and the other piglets received standard weaner diet (CP, 22.5\%; 4: 0, 6: 0 and 12: 0 were not detected in the diet). Within diets, six pigs were inoculated with $E$. coli O149:F4 and the rest of six pigs received saline buffer. At the day prior to challenge and 4 days thereafter, faeces was collected, scored for its consistency, and analyzed for dry matter and bacterial counts. Blood was sampled at the day prior to and 4 days post inoculation (PI), and just before killing. Four pigs (1 per treatment) were killed per day at 6, 7, 8 and 9 days PI. Bile was obtained and mucosa was collected from $10 \%, 50 \%$ and $90 \%$ site of small intestine (SI). Mucosal IgA concentration was lower $(P=0.01)$ in piglets provided with treatment diet, and numerically increased $(P=0.20)$ in $E$. coli challenged piglets. Concentration of IgA decreased distally $(P=0.01)$ from site $10 \%$ length to $50 \%$ and $90 \%$ of SI. Metabolomics profile of the plasma differed between $E$. coli challenged and control pigs, as well as among piglets originated from different litters, but no difference between diets. Challenge with $E$. coli affected faecal and clinical score $(P<0.0001$, only in the first trial) and reduced feed intake $(P=0.02)$ and gain $(P=0.06)$, but the effect of diets was not observed. Neither diets nor inoculation with $E$. coli influenced lymphocyte subset counts in the blood and concentration of immunoglobulins in the bile (IgA) and plasma (IgA, IgM and $\operatorname{IgG}$ ) at any time of measurements. Our results suggest that low-protein diet supplemented with short- and medium chain fatty acids may impair mucosal immune responses of weaned piglets.

1. De Lange CFM, Pluske J, Gong J et al. (2010) Livest Sci 134, 124-134.

2. Rossi R, Pastorelli G, Cannata S et al. (2010) Anim Feed Sci Tech 162, 1-11. 